\title{
Acute Myeloid Leukemia with Gene Mutations
}

National Cancer Institute

\section{Source}

National Cancer Institute. Acute Myeloid Leukemia with Gene Mutations. NCI Thesaurus. Code $C 82430$

A term referring to acute myeloid leukemias associated with gene mutations most often of the FLT3, NPM1, and CEBPA genes. These leukemias may have normal karyotypes or additional chromosomal aberrations. 